\title{
Conditions on a Connection to be a Metric Connection
}

\author{
B. G. Schmidt \\ I. Institut für Theoretische Physik, Universität Hamburg
}

Received July 2, 1972

\begin{abstract}
It is shown that a torsion free linear connection is determined by a metric of given signature if and only if its holonomy group is a subgroup of the orthogonal group corresponding to the signature.
\end{abstract}

\section{§ 1. Introduction}

It is well known that a Riemannian metric $g$ on a manifold $M$ determines uniquely a torsion free linear connection $\Gamma$ on $M$, called the Levi-Civita connection of $g$ [1]. This connection is determined by the condition that parallel transport with respect to $\Gamma$ should preserve the scalar product defined by $g$. The existence and uniqueness of $\Gamma$ can be proved in various ways ${ }^{1}$. With respect to a local coordinate system $\left(x^{i}\right)$ the Christoffel symbols of $\Gamma$ are related to the components of the metric tensor by

$$
g_{k l} \Gamma_{j i}^{l}=\frac{1}{2}\left(g_{k i \mid j}+g_{k j \mid i}-g_{j i \mid k}\right)
$$

which is because of $\Gamma_{k l}^{i}=\Gamma_{l k}^{i}$ equivalent to

$$
g_{h j \mid r}=g_{h i} \Gamma_{j r}^{i}+g_{j l} \Gamma_{h r}^{l} .
$$

The purpose of this paper is to answer the following question: What are the necessary and sufficient conditions for a torsion free connection to be the Levi-Civita connection of a metric?

The most straight forward approach to this problem is to start with the differential equations (2) and write down the integrability conditions for the existence of a solution $g_{i k}$ of $(2)^{2}$. These integrability conditions form a system of functional equations $F^{v}\left(g_{i k}, \Gamma_{k l}^{i}, \Gamma_{k l \mid s_{1} \ldots s_{j}}^{i}\right)=0, v=1,2 \ldots$ whose consistent solvability are necessary and sufficient for the existence of a solution of (2).

${ }^{1}$ A very elementary proof: calculate $\frac{d}{d t}\left(g_{i k}\left(x^{l}(t)\right) a^{i}(t) b^{k}(t)\right)=0$ for $a^{i}(t), b^{k}(t)$ parallel propagated along $x(t)$. Using $\frac{d a^{i}}{d t}+\Gamma_{k l}^{i} \dot{x}^{k} a^{l}=0, \frac{d b^{h}}{d t}+\cdots$ one gets (2).

2 This was done in some unpublished work by Müller zum Hagen. 
Very little insight however is gained by this method into the geometrical meaning of the integrability conditions and the restrictions imposed by them on the connection.

A more geometric approach is the following: Given a connection on a manifold $M$ one can parallel propagate frames. For any path $\tau$ between two points of $M$ parallel transport along $\tau$ defines a linear mapping $L(\tau)$ between the tangent spaces of the two points. This linear map is an isometry if the connection is a Levi-Civita connection.

The holonomy group $\Phi(x)$ of a point $x \in M$ is the group of linear transformations in the tangent space of $x$ defined by parallel propagation along loops starting at $x$. For connections on connected manifolds the holonomy groups of different points are isomorphic.

It is obvious that a connection can only be a Levi-Civita connection of a metric $g$, if the holonomy group is a subgroup of the generalised orthogonal group corresponding to the signature of $g$.

In the next Section I will show that this condition is also sufficient. The basic idea is to extend orthonormal frames determined by the holonomy group at one point onto the whole manifold by parallel transport.

In the last section some comments are made on the relations between the infinitesimal holonomy group, which can be calculated from the Riemann tensor and its derivatives, and the holonomy group.

\section{$\S 2$. Proof of the Theorem}

Theorem. Let $\Gamma$ be a torsion free connection on a connected manifold $M$ whose holonomy group $\Phi$ keeps a non degenerate quadratic form $g_{0}$ invariant. Then $\Gamma$ is the Levi-Civita connection of a metric which has the same signature as $g_{0}$.

Proof. The holonomy group $\Phi(x)$ of a point $x \in M$ keeps $g_{0}$ invariant. Therefore we can define at $x$ a scalar product $g_{x}(X, Y)$ which is invariant under $\Phi(x)$. For any $y \in M$ there is a path $\tau$ joining $x$ and $y$. An unique scalar product $g_{y}(X, Y)$ is defined at $y \in M$ by the condition that $L(\tau)$ is an isometry. If $\sigma$ is any other path from $x$ to $y$ then $L(\tau) L\left(\sigma^{-1}\right)$ is an element of $\Phi(x)$, hence an isometry. This implies that $L(\sigma)$ is an isometry. Therefore $g_{y}$ is independent of the particular path joining $x$ and $y$. In this way we define a metric on $M$ which is $C^{\infty}$ if $\Gamma$ is $C^{\infty}$.

Now it is easy to see that parallel transport with respect to $\Gamma$ preserves $g(X, Y)$ defined above: Let $\tau$ be a path joining two arbitrary points $y$ and $z$. Then there is a path $\sigma$ from $x$ to $y$ and a path $\lambda$ from $z$ to $x$. This implies $L(\sigma) L(\tau) L(\lambda) \in \Phi(x)$. From the definition of $g$ we know that $L(\sigma)$ and $L(\lambda)$ are isometries, hence $L(\tau)$ is an isometry. 
Now we have constructed a metric $g$ on $M$ with the property that parallel transport with respect to $\Gamma$ preserves the scalar product $g$. On the other hand we know from $\S 1$ that the Levi-Civita connection of $g$ is the only connection having this property, hence $\Gamma$ is the LeviCivita connection of $g$ and the proof is completed.

A Levi-Civita connection determines the metric not uniquely. Obviously $g$ and $\alpha g$ have the same Levi-Civita connection if $\alpha$ is constant.

The theorem above shows that every $g_{0}$ invariant under $\Phi(x)$ defines a metric whose connection is $\Gamma$. To get all metrics we have to calculate all non degenerate quadratic forms invariant under $\Phi(x)$.

Suppose for example that there are two complementary subspaces of $T_{x}(M)$ invariant under $\Phi(x)$ and orthogonal with respect to $g_{0}$. Then $\alpha g_{1}+\beta g_{2}$ is also invariant under $\Phi(x)$ if $g_{1}, g_{2}$ are the restrictions of $g_{0}$ on the subspaces ${ }^{3}$.

This situation however occures only in a very restricted class of spaces, because it implies that the Riemannian space is at least locally the direct sum of two spaces [1]. Hence a Levi-Civita connection in general will determine the metric up to a constant conformal factor. To enter the classification of the particular cases in which there are more metrics is beyond the purpose of this paper.

\section{§ 3. Determination of the Holonomy Group of a Connection}

The holonomy group decides whether a connection is a Levi-Civita connection or not. How can we find the holonomy group of a connection and the quadratic forms invariant under it?

Let us first consider a connection on a simply connected manifold. In this case $\Phi(x)$ is a connected Lie subgroup of the group of linear transformations in the tangent space of $x$. Therefore $\Phi(x)$ is uniquely determined by its Lie algebra $\underline{\Phi(x)}$ and the metric $g_{x}(X, Y)$ is invariant under $\Phi(x)$ if and only if

$$
g_{x}(A(X), Y)+g_{x}(X, A(Y))=0
$$

holds for any $A \in \Phi(x)$. Hence if $M$ is simply connected we can reformulate the assumptions of the Theorem in $\S 2$ as assumptions on $\Phi$.

Suppose for example $\Gamma$ were analytic. Then it is shown in [1] that the linear maps

$$
\nabla^{k} R\left(X, Y ; V_{1} \ldots V_{k}\right), X, Y, V_{i} \in T_{x}(M)
$$

or equivalently the matrices

$$
R_{k l m ; s_{1} \ldots s_{k}}^{i} \xi^{l} \eta^{m} v^{s_{1}} \ldots v^{s_{k}}
$$

\footnotetext{
${ }^{3}$ It is still possible that some of the metrics $\alpha g_{1}+\beta g_{2}$ are isometric!
} 
span the Lie algebra of the holonomy group. Therefore we can calculate $\Phi(x)$ from the Riemann tensor and its derivatives if the connection is analytic.

For $C^{\infty}$ connections the linear maps (4) form still a Lie algebra $\Phi^{\prime}(x)$ and the group generated by it is called the infinitesimal holonomy group $\Phi^{\prime}(x)$. In general however $\Phi^{\prime}$ is only a subgroup of $\Phi$. Consider as an example a connection with the property that the Riemann tensor and all its derivatives vanish at one point $x$ but not in an open set containing $x$. Then $\Phi^{\prime}(x)$ is trivial but $\Phi(x)$ not.

In [1] it is proved that $\operatorname{dim} \Phi^{\prime}=$ const implies $\Phi^{\prime}(x)=\Phi(x)$. Hence the Riemann tensor and its derivatives at one point determine $\Phi(x)$ in this case.

The following example serves as a counter example to two conjectures:

(A) There is always a point $x$ in which $\Phi^{\prime}(x)=\Phi(x)$ holds.

(B) If any point has a neighbourhood such that $\Gamma$ restricted to this neighbourhood is a Levi-Civita connection, then $\Gamma$ is a Levi-Civita connection.

Consider the following three parts of $R^{2}$, where $(t, x)$ is a global coordinate system:

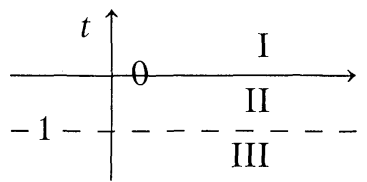

$$
\begin{aligned}
\mathrm{I} & =\{(x, t) ; t>0\} \\
\mathrm{II} & =\{(x, t) ;-1<t<0\} \\
\mathrm{III} & =\{(x, t) ; t<-1\} .
\end{aligned}
$$

We define Lorentz metrics in I and III by

$$
d s_{\mathrm{I}}^{2}=-d t^{2}+R^{2}(t) d x^{2}, \quad d s_{\mathrm{III}}^{2}=-d t^{2}+S^{2}(t) d x^{2} .
$$

The only non vanishing Christoffel symbols are

$$
R \dot{R}, \frac{\dot{R}}{R} \text { for I, } S \dot{S}, \frac{\dot{S}}{S} \text { for III . }
$$

Suppose $R(t)$ and $S(t)$ are $C^{\infty}$ functions satisfying

$$
\begin{array}{ll}
\lim _{t \rightarrow 0} R(t)=1 & \lim _{t \rightarrow 0} \frac{d^{n} R}{d t^{n}}=0 \\
\lim _{t \rightarrow-1} S(t)=a \neq 1 & \lim _{t \rightarrow-1} \frac{d^{n} S}{d t^{n}}=0
\end{array} \quad n=1,2, \ldots .
$$

We define a connection on $R^{2}$ by $\Gamma_{k l}^{i}=0$ on $-1 \leqq t \leqq 0$ and the LeviCivita connections of the metrics (6) on I and III. 
Let us now check whether this connection is a Levi-Civita connection: It is clear that $\alpha\left(-d t^{2}+R^{2} d x^{2}\right)$ is the most general metric on I. For any constant $\alpha$ there is an unique continuous extension of this metric on II, namely $\alpha\left(-d t^{2}+d x^{2}\right)$, such that $\Gamma$ is the Levi-Civita connection on I $U$ II. Similar $\beta\left(-d t^{2}+S^{2} d x^{2}\right)$ is the general metric on III and its unique extension to get the right connection on II is $\beta\left(-d t^{2}+a^{2} d x^{2}\right)$. Therefore we get a contradiction on II because $a^{2} \neq 1$. The connection we defined is no Levi-Civita connection and it contradicts (A) and (B).

Despite of the fact that in general we can not calculate the holonomy group from the Riemann tensor and its derivatives one should keep the following in mind: The set of points with $\operatorname{dim} \Phi^{\prime}(x) \geqq k$ is always open [1]. Therefore one can find open submanifolds on which $\Gamma$ determines possible metrics. For practical purposes - if this problem arises at all one can consider these open submanifolds separately and then try to join the metrics.

Let us finally turn to connections on manifolds which are not simply connected. Then the holonomy group is not necessarily connected. If $\Phi_{0}$ is the connected component of $\Phi$ then $\Phi / \Phi_{0}$ is a subgroup of $\pi_{1}(M)$, the first homotopy group of $M$.

The connection $\Gamma$ on $M$ defines uniquely a connection $\tilde{\Gamma}$ on $\tilde{M}$, the universal covering space, by the condition that the projection $\pi: \tilde{M} \rightarrow M$ is an affine isometry for sufficient small open sets in $\tilde{M}$. The holonomy group of $\tilde{\Gamma}$ is $\Phi_{0}$. Hence $\tilde{\Gamma}$ is a Levi-Civita connection if this is the case for $\Gamma$.

Suppose conversely that $\tilde{\Gamma}$ is a Levi-Civita connection on $\tilde{M}$. Then $\Gamma$ is a Levi-Civita connection if and only if $\Phi / \Phi_{0}$, which acts as a transformation group on $\tilde{M}$, is a group of isometries of the metric on $\tilde{M}$.

An example of a connection on $S^{1}$ which is only a Levi-Civita connection on the universal covering space is the following: $d s^{2}=e^{x} d x^{2}$ is a metric on $R^{1}$. The map $x \rightarrow x+1$ is an affine transformation but not an isometry. By identification we get a connection with the desired property on $S^{1}$.

\section{References}

1. Kobayashi, S., Nomizu, K.: Foundations of differential geometry, Vol. 1. New York: Interscience 1963.

B. G. Schmidt

I. Institut für Theoretische Physik der Universität

D-2000 Hamburg 36, Jungiusstr. 9

Federal Republic of Germany 
